\title{
Enrolling HIV-positive adolescents in mental health research: A case study reflecting on legal and ethical complexities
}

\author{
N Woollett, ${ }^{1}$ MA (Psychology, Art Therapy); J Peter, ${ }^{2}$ SC, BCom, LLB, LLM; L Cluver, ${ }^{3}$ DPhil; H Brahmbhatt, ${ }^{4}$ MPH, PhD \\ ${ }^{1}$ Department of Paediatrics and School of Public Health, Faculty of Health Sciences, University of the Witwatersrand, Johannesburg; \\ and Wits Reproductive Health and HIV Institute, Johannesburg, South Africa \\ ${ }^{2}$ Johannesburg Bar, South Africa; and New South Wales Bar, Australia \\ ${ }^{3}$ Centre for Evidence-Based Intervention, Department of Social Policy and Intervention, Oxford University, UK \\ ${ }^{4}$ Department of Population, Reproductive and Family Health, Bloomberg School of Public Health, Johns Hopkins University, Baltimore, USA; \\ and Wits Reproductive Health and HIV Institute, University of the Witwatersrand, Johannesburg, South Africa
}

Corresponding author: N Woollett (woollettn@gmail.com)

Background. Adolescents living with HIV are an emerging group in the global HIV/AIDS epidemic. Mental health in this population affects HIV care, treatment, consequential morbidity and secondary transmission. There is a paucity of research regarding these youth in South Africa (SA), partly because section 71 of the National Health Act of 2003 (NHA) requires parental or guardian's consent.

Objective. To explore legal and ethical issues related to conducting adolescent mental health research in SA.

Methods. After obtaining a High Court order permitting research on minors aged $<18$ years without prior parental or guardian's consent, we used qualitative and quantitative methods to interview adolescents in five clinics serving HIV-positive adolescents in Johannesburg. Results. Our study enrolled 343 participants; 74\% were orphaned and did not have legal guardians, 27\% were symptomatic for depression, anxiety or post-traumatic stress disorder, $24 \%$ were suicidal, and almost $90 \%$ did not feel that they belonged in the family with which they lived. Without court intervention, most of the participants could not have participated in this research because parental consent was impossible to obtain. This case study argues for exceptions to the parental consent requirement, which excludes orphaned and vulnerable children and youth from research.

Conclusions. Recommendations are made to promote ethical integrity in conducting mental health research with adolescents. A balance is needed between protecting adolescents from exploitation and permitting access to benefits of research. Requiring parental consent for all research does not necessarily give effect to policy. For the vast majority of SA HIV-positive adolescents, parental consent is not possible. Section 71 of the NHA ought to be amended to facilitate valuable and necessary research concerning HIV-positive orphan children and adolescents.

S Afr Med J 2017;107(8):679-683. DOI:10.7196/SAMJ.2017.v107i8.12409

\section{The adolescent burden of HIV}

Adolescents living with HIV are an emerging group in the global HIV/AIDS epidemic. In 2012, there were 2.1 million HIV-positive adolescents in low- and middle-income countries (LMICs); globally there were 3.2 million HIV-positive children aged $<15$ years, $90 \%$ of whom were living in sub-Saharan Africa. ${ }^{[1,2]}$ Nearly one-sixth of all new HIV infections are in adolescents aged 15 - 19 years, ${ }^{[3]}$ making this group the most vulnerable incident infection population in South Africa (SA). ${ }^{[4]}$ In addition, owing to significant improvements in accessing antiretroviral therapy, children born with HIV are growing into adolescence in large numbers, especially in LMICs. ${ }^{[5,6]}$

Worldwide, adolescents are the only age group in which AIDSrelated deaths are not decreasing. ${ }^{[3]}$ In 2013, there were $>9500$ deaths of HIV-infected adolescents in SA. ${ }^{[7]}$ Local studies contrasting adolescents with adults indicate lower retention in care and viral suppression among youth. ${ }^{[8,9]}$ The cohort of sexually active, HIVpositive and viraemic youth makes a significant impact on HIV transmission rates, warranting consideration of public health investment. Engagement of adolescents is critical to a meaningful HIV response.
Approximately 15 million children in sub-Saharan Africa have lost one or both parents to AIDS, 2.5 million of them in SA. ${ }^{[10]} \mathrm{An}$ orphanhood epidemic has matured alongside the HIV epidemic, with high rates of adolescent orphans. In 2012, the overall national level of orphans aged $<18$ years was $16.9 \%$ (maternal $4.4 \%$, paternal 9.3\%, double 3.2\%). ${ }^{[4]}$ Families of HIV-positive children and adolescents have high levels of mobility and migration, with inconsistent guardianship, care and supervision. ${ }^{[11-13]}$ Typically when biological parents die, their children are taken into care by extended family or friends. Unless a child is placed in formal institutional care, the formal appointment of a legal guardian is extremely rare. Legal guardianship is often not meaningful to black African families. ${ }^{[14]}$

\section{Rates of mental health problems in adolescents}

In both developed and less-developed countries, almost 50\% of people living with HIV/AIDS have a diagnosable mental disorder in some instances a rate three times higher than that in the general population. ${ }^{[15-18]}$ Reasons for this include premorbid mental conditions, the effect of HIV on the central nervous system, the psychological 
impact of living with HIV/AIDS, side-effects of medication, social stigma and discrimination. ${ }^{[15]}$ Mental health disorders occur with increased frequency among HIV-positive children and adolescents in developed contexts. ${ }^{[12,19,20]}$ In LMICs there is a paucity of research on this population and mental health outcomes. Studies in Western countries, measuring the association between HIV and mental health outcomes in adolescents, are not always comparable with black African populations, where adolescents are subjected to multiple additional risks and vulnerabilities. Poor mental health is strongly related to other health and development concerns in young people - lower educational achievements, neurocognitive delay, substance abuse, violence, and poor reproductive and sexual health. ${ }^{[21-25]}$ Examination of the impact of HIV on mental health outcomes in adolescents locally is critical; mental health is likely to affect HIV care and treatment, the resulting adult burden of disease and secondary transmission.

\section{Difficulties with undertaking research on this group}

SA legislation requiring parental or guardian's consent presents a significant barrier to research on HIV-positive adolescents aged $<18$ years. Using a study of mental health outcomes of HIV-positive adolescents in Johannesburg as a case study, we explore legal and ethical issues related to conducting adolescent mental health research.

\section{The legal framework - the National Health Act of 2003 (NHA) ${ }^{[26]}$}

Prior to March 2012, the SA framework governing research permitted children $(<18$ years) to consent independently to take part in research. ${ }^{[27]}$ Where the research posed minimal risk and no community objection was anticipated, national ethical guidelines permitted an exemption from parental or guardian's consent for participation in health research by older children. ${ }^{[1,28]}$

On 1 March 2012, section 71 of the NHA came into effect, which has four prerequisites for research or experimentation on a minor for therapeutic purposes: (i) the best interests of the minor; (ii) conformity with the manner and conditions prescribed; (iii) consent of the parent or guardian; and (iv) consent of the minor where capable of understanding. Non-therapeutic research and experimentation require additional ministerial consent. ${ }^{[29]}$

On 19 September 2014, the Regulations Relating to Research with Human Participants were promulgated, completing the new legal framework for regulating health research established by the NHA. ${ }^{[30]}$ These regulations address three general issues relating to children. First, minors are considered a vulnerable population. Health research ethics committees (HRECs) are required to balance child protection and research facilitation and pay special attention to protocols, recognising children as deserving beneficiaries of research outcomes. ${ }^{[31]}$ Second, the participation of minors must be scientifically indispensable to the study design. ${ }^{[31]}$ Third, minors can only participate in research when they will be exposed to particularly low levels of risk, an approach that corresponds with the risk categories described in national ethical guidelines. ${ }^{[30]}$

The regulations define 'therapeutic research' as research that holds out the prospect of direct benefit to the participant. Neither the NHA nor the regulations defines 'research'.

\section{Impractical for researchers: Woollett case study}

Our case study (a description of the mental health of HIV-positive adolescents accessing care in Johannesburg) highlights the legal process undertaken to facilitate research on vulnerable SA adolescents. It could not be done with parental or guardian's consent, as the proposed participants would be orphans without legal guardians. Approval was initially refused by the University of the Witwatersrand medical HREC. In July 2013, the principal investigator applied to the High Court, Johannesburg, in its capacity as upper guardian of minors, for consent for the research. The application was served on the facility managers of the five clinics concerned, the provincial and district research committees, the Chris Hani Baragwanath and Charlotte Maxeke academic hospital research committees, the provincial health MEC and the health minister. Upon the order being granted, the university HREC granted unconditional approval of the study (M130258), as did the other research committees. This process took 8 months and without pro bono legal assistance would have been impossible.

The study integrated qualitative and quantitative methods in design and implementation. It took place in five clinics serving HIV-positive adolescents in Johannesburg. A survey was undertaken of $343 \mathrm{HIV}$-positive adolescents aged 13 - 19 years. It contained standard assessments for depression, anxiety, post-traumatic stress disorder (PTSD) and suicide and captured HIV, sexual reproductive health, adherence, disclosure and demographic information.

Of the 343 participants, $74 \%$ were orphaned and did not have legal guardians. Most were probably perinatally infected. Twenty-seven percent were symptomatic for depression, anxiety or PTSD, 24\% indicated signs of suicidality (excluding 17 adolescents excluded for active suicidality), almost $90 \%$ did not feel that they belonged within the family fostering them, $90 \%$ did not receive praise from those they lived with, and $85 \%$ were not given the same things as other children in the home. But for the High Court order, most of the sample would probably not have participated in this research.

\section{Issues related to parental consent}

Much debate has taken place over allowing children to consent to health research. ${ }^{[14,27,32,33]}$ SA HIV research is still abundant, and the greatest burden of disease has moved to vulnerable groups, including adolescents. Research on adolescents is critical, but inhibited by requiring parental consent.

\section{Exclusion of most orphaned and vulnerable children and youth (OVCY)}

The adverse outcomes of being orphaned include loss of effective guidance and supervision, inconsistent care, loss of educational opportunities, impoverishment, increased sexual vulnerability, high rates of risk-taking, psychological distress and significant mental health problems. ${ }^{[19]}$ There is a higher prevalence of HIV infection among orphans due to AIDS causes than among non-orphans and orphans of non-AIDS causes, together with earlier sexual debut, an increased risk of intergenerational sex, and increased family violence and exploitation. ${ }^{[10,34-37]}$

OVCY are increasingly recognised as a special population for HIV risk and transmission. ${ }^{[35]}$ OVCY, child-headed households and children and youth without an official guardian are a unique and contemporary issue placing many SA institutions (including government) under tremendous pressure. Innovation and responsiveness are key factors to counter these challenges. This vulnerable group can ill afford to be neglected by research, which could assist in HIV prevention and yield effective treatment interventions.

Parental consent is impossible for minors not living with, or having access to, their parents, and for orphans, who comprise a significant proportion of HIV-positive children. Section 71 has the effect of impeding research on child-headed households and OVCY. 


\section{Inconsistent and confusing legal policy}

Section 129 of the Children's Act of $2005^{[38]}$ permits independent autonomous consent to general medical treatment by a child aged $>12$ years, including HIV counselling and testing. The Choice on Termination of Pregnancy Act of $1996^{[39]}$ provides for autonomous decision-making by a pregnant child with no minimum age requirement. Policy recognises child and adolescent capacity to make decisions concerning themselves. An adolescent child may independently consent to an HIV test and receive medical treatment, but cannot consent to the observation of such for research to improve the quality of, or access to, treatment. Excluding autonomous participation in observational research of a low-risk nature undermines this policy.

\section{Inadvertent harm or silence to voices that most need to be heard}

Adolescents may opt not to seek care because they want to avoid telling their parents about their health problems and sexual activity. ${ }^{[1]}$ The Children's Act ${ }^{[38]}$ facilitates confidentiality from parents, avoiding stigma, discrimination and potential punishment. Requiring parental consent for research removes confidentiality. Children who are unable to obtain parental consent to participate are often those most in need of mental health information and resources. Requiring parental consent excludes their experiences and needs from research findings. ${ }^{[40]}$

Research identifying harm to children may expose the parents and caretakers as the perpetrators of such harm. Parental consent in these circumstances is highly likely to be refused and the harm undetected. SA prevalence rates for abuse in adolescents are $56.3 \%$ for lifetime physical abuse, 35.5\% for lifetime emotional abuse and 9\% for lifetime sexual abuse. ${ }^{[24]}$ Historically, a requirement for parental consent in all cases would have precluded the ground-breaking research by radiologists correlating paediatric head trauma with long-bone fractures identifying child abuse. ${ }^{[41]}$

\section{Requiring parental consent may be inconsistent with principles of justice, inclusiveness and autonomy}

Requiring parental consent implicitly assumes that parents' ability to understand research and assess risk is always superior to their children's. Parents' opinions are often informed by their own experience of adolescence rather than the realities of their children. ${ }^{[40]}$ Mental health is highly stigmatised, difficult to communicate and often not discussed in families.

Most school-aged children are capable of meaningful assent for participation in many types of research studies. Adolescents from 14 years may be as capable as adults of making competent decisions about research participation, according to more stringent legal standards of competency. ${ }^{[42]} \mathrm{SA}$ adolescents view participation in research, particularly HIV vaccine trials, to be beneficial and would participate, their reasons ranging from altruism to direct benefit. ${ }^{[32,43]}$ Adolescents report marginal levels of distress when self-reporting violence and suicidality. ${ }^{[44,45]}$ In our study, the overwhelming majority of adolescents expressed relief and gratitude in being able to tell their story and did not report experiencing the interview as stressful echoing findings in other studies of HIV-affected children. ${ }^{[46-48]}$

The African Charter on the Rights and Responsibilities of the Child recognises rights to enjoy the best attainable state of physical, mental and spiritual health and scientific research. The right to life and the right to access to healthcare rank among the most fundamental of the human rights guaranteed by international human rights. ${ }^{[49]}$ Excluding adolescent children from health research infringes on their constitutional rights to both basic healthcare and access to healthcare services. ${ }^{[50]}$

\section{Recommendations for mental health research with adolescents arising from this study}

To promote ethical integrity, the following were implemented and are examples that may be helpful to other studies:

- A community advisory board that can advise on all aspects of the research, including proposal, protocol, survey, informed consent and a dissemination plan, can ensure youth-friendliness and relevance if constituted by adolescents who utilise services. This is effective in addressing complex health disparities and facilitates local acceptability of the research practices.

- Research staff need a balance of research, community and clinical experience. Lay counsellors, with experience in the clinics managing issues of adherence, disclosure, stigma and HIV counselling, and additional training that emphasises adolescent rights, the distinction between researchers and counsellors, modelling interview techniques, with practice sessions on dealing with informed consent processes, etc. are recommended. Staff also need weekly debriefing and supervision from a registered therapist.

- Consent procedures disclosed that confidentiality may be broken by the mandatory reporting requirements relating to suspected illtreatment, abuse or neglect of children (section 42 of the Children's Act, ${ }^{[38]}$ section 4 of the Prevention of Family Violence Act of $1993,{ }^{[51]}$ and sexual offences against children (section 54 of the Criminal Law (Sexual Offences and Related Matters) Amendment Act of $\left.2007^{[52]}\right)$. Study staff need training to identify reporting obligations, and understand and manage the processes triggered.

- Researchers must be mindful that there is evidence that compared with adults, adolescents are less likely to spontaneously consider risks and benefits, are less likely to evaluate long-term consequences of decisions, are more likely to place weight on benefit than risk, are more likely to be short-term focused, and are less likely to recognise the vested interests of others. ${ }^{[43]}$ Recognition of immature decision-making is therefore necessary, and researchers should try to enhance adolescent understanding to the fullest extent possible. To assess understanding, our informed consent contained both multiple-choice questions and open-ended probing questions.

- The latent power dynamic between researchers and adolescent participants must be managed. Contextually, obedience to and respect for adults are values strongly emphasised; children seldom speak up or voice their opinions to adults. ${ }^{[53]}$ Time needs to be provided for the adolescents to acquire trust in the researchers and the research process. Research counsellors were able to speak all the local languages, increasing their ability to answer all questions posed and alleviate potential anxiety of the participants.

- Authenticity in child participatory research is crucial: without it, the validity of what is reported is inevitably questionable and, at the level of tokenism, it is fundamentally unethical. ${ }^{[33]}$ Research utilising non-verbal methods, such as drawings, can offer research subjects active participation in the research process, authenticating their voice through their engagement, offering more developmentally appropriate means of accessing data, diminishing stress in the child/adolescent-adult interaction, and providing a more comfortable method of engagement than language. ${ }^{[3-56]}$

- Engaging youth in research can be facilitated with the use of mobile and computer devices, known to be appealing. Data were collected on tablet computers to improve reporting of sensitive questions. ${ }^{[57,58]}$

- HRECs reviewing adolescent protocols should consult with one another to facilitate uniformity in response to similar research protocols. ${ }^{[43]}$ 


\section{Discussion}

Evidence regarding the mental health of HIV-positive adolescents is fundamental to inform policy. In this case study, $27 \%$ of HIVpositive adolescents accessing treatment were experiencing common mental health problems that were previously unrecognised and could be treated in the public health system. But for a court order, this evidence would not have been available. Vulnerable groups such as adolescents are typically the last to benefit from research, and often need it the most.

Laws such as the NHA were developed to safeguard children, and in many cases do. Section 71 has the policy objective to protect them. Where the parental consent requirement is inappropriate or impossible, it operates to exclude children from research, further increasing their vulnerability and failing its policy objective. Research ethics legislation is vital and welcomed, but a balance is needed between protection from exploitation and access to benefits of research. Excessive regulation to protect adolescents from exploitative research may impede on their human rights and autonomy as individuals and circumvents research being undertaken to improve access to care and treatment and reform national policy. ${ }^{[27]}$ It is also likely to delay their access to any improvement based on adult research undertaken. ${ }^{[59]}$ Provision should be made for rigorous review by HRECs and promoting clear communication to adolescents rather than restrictions that reduce research participation. ${ }^{[30]}$ The NHA and its regulations ought to take into account the social contexts of SA adolescents. A rights-based approach is necessary in managing these tensions. ${ }^{[60]}$ As highlighted by Mann (in Kirby ${ }^{[49]}$ ), "health and human rights are complementary approaches to the central problem of advancing human well-being?

Failure to address mental health problems in terms of prevention and treatment, including developmental and intellectual disorders, in childhood and adolescents in low-resource settings is a public health issue with far-reaching consequences. A substantial proportion of adult mental health problems originate early in life, and such failure has long-lasting effects beyond childhood and adolescence ${ }^{[60]}$ and impedes the achievement of basic development goals in LMICs.

A commitment to evidence-based healthcare requires more, not less, effort to encourage research and clear evaluation of services. Service and research entities need to assess whether their services/ interventions are helping children/adolescents and how they can be improved. Excluding the most vulnerable adolescents risks biasing the research capacity to assess their needs and services to them.

These findings suggest the importance of considering an amendment to section 71 of the NHA, in order to facilitate valuable and necessary research concerning HIV-positive and orphaned children. A mechanism ought to be introduced to permit research on children where parental or guardians' consent is either not practicable or inappropriate. 'Research' ought to be defined. A distinction should be made to cater for HRECs to allow a dispensation from parental consent for classes of research that present minimal risk of harm to child participants, and an age threshold should be introduced for children to give autonomous consent. Further practical examples of how to address the challenges related to this work in diverse contexts are needed in order to build a consensus on best practices.

\section{Conclusions}

Ethical and sound health research is essential to promoting the health of highly vulnerable HIV-positive and affected adolescents. If research results are to be targeted to adolescents, it is essential that adolescents be included so that study outcomes are relevant to them. Healthcare policy is best improved based on the evidence to meet the mental health needs of this population more effectively.
Requiring parental consent for particular research does not necessarily give effect to policy. For the vast majority of HIV-positive adolescents, such consent is simply impossible. The result is that the most vulnerable are excluded from research that may yield great benefit. Resort to the High Court is practically unworkable and legislative amendment is urgently required.

Acknowledgements. We thank the participants for sharing their stories. We acknowledge the impressive work of the research counsellors (Thamsanqa Jabavu, Nombulelo Shezi, Linda Mazibuko, Princess Mbatha, Tebogo Moloi, Honey Nyapoli, Bafana Gxubane, Shenaaz Randeria and Relebohile Maleka), both in their capacity as counsellors and for their enthusiasm in engaging in this research. We are grateful to the national and provincial departments of health for allowing access to the patients and facilities in Johannesburg. Thanks also to Ms Justine Michel of Fluxmans Attorneys, Johannesburg, for legal assistance.

Author contributions. NW conceived and undertook the study and drafted the manuscript for publication; JP secured the court order to undertake the study and contributed to the drafting and editing of the manuscript; HB and LC provided overall guidance on the protocol development and design of the study; and HB provided overall oversight of the study. All authors read and approved the final manuscript.

Funding. None.

Conflicts of interest. None.

1. World Health Organization. HIV and Adolescents: Guidance for HIV Testing and Counselling and Care for Adolescents Living with HIV: Recommendations for a Public Health Approach and Considerations for Policy-makers and Managers. Geneva: WHO, 2013. http://apps.who.int/iris/ Considerations for Policy-makers and $\mathrm{M}$ )

2. Joint United Nations Programme on HIV/AIDS. The Gap Report. Geneva: UNAIDS, 2014. http:// . Joint United Nations Programme on HIV/AIDS. The Gap Report. Geneva: UNAIDS, 2014. http./
www.unaids.org/en/resources/campaigns/2014/2014gapreport/gapreport (accessed 5 April 2017). 3. United Nations Children's Fund. Towards an AIDS-free Generation - Children and AIDS: Sixth Stocktaking United Nations Children's Fund. Towards an AIDS-free Generation - Children and AIDS: Sixth Stocktaking
Report, 2013. New York: UNICEF, 2013. https://www.unicef.org/publications/index_70986.html (accessed 5 April 2017)

4. Shisana O, Rehle T, Simbayi LC, et al. South African National HIV Prevalence, Incidence and Behaviour Survey. Pretoria: HSRC, 2012. http://www.hsrc.ac.za/en/research-data/view/6871 (accessed 5 April 2017).

5. Ferrand RA, Corbett EL, Wood R, et al. AIDS among older children and adolescents in southern Africa: Projecting the time course and magnitude of the epidemic. AIDS 2009;23(15):2039-2042. https://doi.org/10.1097/qad.0b013e32833016ce

6. Idele P, Gillespie A, Porth T, et al. Epidemiology of HIV and AIDS among adolescents: Current status, nequities, and data gaps. J Acquir Immune Defic Syndr 2014;66(Suppl 2):S144-S153. https://doi. org/10.1097/qai.0000000000000176

7. Joint United Nations Programme on HIV/AIDS. HIV Estimates. UNAIDS, 2013. http://www.unaids. org/sites/default/files/media_asset/UNAIDS_Global_Report_2013_en_1.pdf (accessed 5 April 2017).

8. Zanoni BC, Archary M, Buchan S, Katz IT, Haberer JE. Systematic review and meta-analysis of the adolescent HIV continuum of care in South Africa: The cresting wave. BMJ Glob Health 2016;1(3):1adolescent HIV continuum of care in South Afri

10. https://doi.org/10.1136/bmjgh-2015-000004 Organization. Global HIV/AIDS Response: Epidemic Update and Health Sector Progress Towards Universal Access: Progress Report 2011. WHO, 2011. http://www.who.int/hiv/pub/progress_ report2011/hiv_full_report_2011.pdf (accessed 5 April 2017)

10. Sharp C, Jardin C, Marais L, Boivin M. Orphanhood by AIDS-related causes and child mental health: A developmental psychopathology approach. J HIV AIDS 2015;1(3):1-19. https://doi, org $/ 10.16966 / 2380-5536.114$

11. United Nations Children's Fund. South Africa's Children - a Review of Equity and Child Rights. Pretoria: South African Human Rights Commission \& UNICEF, 2011. https://www.unicef.org/ southafrica/SAF_resources_factschildrens11.pdf (accessed 5 April 2017).

12. Lowenthal ED, Bakeera-Kitaka S, Marukutira T, Chapman J, Goldrath K, Ferrand RA. Perinatally acquired HIV infection in adolescents from sub-Saharan Africa: A review of emerging challenges. acquired HIV infection in adolescents from sub-Saharan Africa: A review of emerg

13. Ford K, Hosegood V. AIDS mortality and the mobility of children in KwaZulu-Natal, South Africa. Ford K, Hosegood V. AIDS mortality and the mobility of children in Kw
Demography 2005;42(4):757-768. https://doi.org/10.1353/dem.2005.0029

14. Strode AE. Using the concept of parental responsibilities and rights to identify adults able to provide proxy consent to child research in South Africa. S Afr J Bioeth Law 2011;4(2):69-73.

15. Freeman M, Nkomo N, Kafaar Z, Kelly K. Factors associated with prevalence of mental disorder in people living with HIV/AIDS in South Africa. AIDS Care 2007;19(10):1201-1209. https://doi. org/10.1080/09540120701426482

16. Myer L, Seedat S, Stein DJ, Moomal H, Williams DR. The mental health impact of AIDS-related mortality in South Africa: A national study. J Epidemiol Community Health 2009;63(4):293-298. https://doi.org/10.1136/jech.2008.080861

17. Lazarus R, Freeman M. Primary-Level Mental Health Care for Common Mental Disorder in ResourcePoor Settings: Models \& Practice. A Literature Review. Pretoria: Medical Research Council, 2009.

18. Thom R. Common mental disorders in people living with HIV/AIDS: Clinical overview. South Afr J HIV Med 2009;10(3):8-13.

19. Williams SL, Williams DR, Stein DJ, Seedat S, Jackson PB, Moomal H. Multiple traumatic events and psychological distress: The South Africa Stress and Health Study. J Trauma Stress 2007;20(5):845-855. psychological distress: The South

20. Gadow KD, Angelidou K, Chernoff M, et al. Longitudinal study of emerging mental health concerns in youth perinatally infected with HIV and peer comparisons. J Dev Behav Pediatr 2012;33(6):456-468. https://doi.org/10.1097/dbp.0b013e31825b8482 
21. Domek GJ. Social consequences of antiretroviral therapy: Preparing for the unexpected futures of HIVpositive children. Lancet 2006;367(9519):1367-1369. https://doi.org/10.1016/s0140-6736(06)68584-x 22. Domek GJ. Facing adolescence and adulthood: The importance of mental health care in the global 2. Domek GJ. Facing adolescence and adulthood: The importance of mental health care in the global
pediatric AIDS epidemic. J Dev Behav Pediatr 2009;30(2):147-150. https://doi.org/10.1097/ pediatric AIDS epide
dbp.0b013e318196b0cc

23. Sherr L, Mueller J. Where is the evidence base? Mental health issues surrounding bereavement and HIV in children. J Public Ment Health 2009;7(4):31-39. https://doi.org/10.1108/17465729200800027

24. Sherr L, Croome N, Castaneda KP, Bradshaw K, Romero RH. Developmental challenges in HIV infected children - an updated systematic review. Child Youth Serv Rev 2014;45:74-89. https://doi. org/10.1016/j.childyouth.2014.03.04

25. Meinck F, Cluver LD, Boyes ME, Loening-Voysey H. Physical, emotional and sexual adolescent abuse victimisation in South Africa: Prevalence, incidence, perpetrators and locations. J Epidemiol Comm Health 2016;70(9):910-916. https://doi.org/10.1136/jech-2015-205860

26. South Africa. National Health Act No. 61 of 2003.

27. Zuch M, Mason-Jones AJ, Mathews C, Henley L. Changes to the law on consent in South Africa: Implications for school-based adolescent sexual and reproductive health research. BMC Int Health Hum Rights 2012;12(1):3-8. https://doi.org/10.1186/1472-698x-12-3

28. National Department of Health, South Africa. Ethics in Health Research: Principles, Structures and Processes. Pretoria: $\mathrm{NDoH}, 2004$.

29. National Department of Health, South Africa. Proclamation No. 11. Government Gazette No. 35081, 27 February 2012.

30. Strode AE, Slack CM. Child research in South Africa: How do the new regulations help? S Afr Med J 2015;105(11):899-900. https://doi.org/10.7196/samj.2015.v105i11.9838

31. South Africa. Regulations Relating to Research with Human Participants. Government Gazette No. R719, 19 September 2014

32. Jaspan HB, Berwick JR, Myer L, et al. Adolescent HIV prevalence, sexual risk, and willingness to participate in HIV vaccine trials. J Adolesc Health 2006;39(5):642-648. https://doi.org/10.1016/j. jadohealth.2006.05.016

33. Fisher $\mathrm{CB}$, Hoagwood K, Boyce $\mathrm{C}$, et al. Research ethics for mental health science involving ethnic minority children and youths. Am Psychol 2002;57(12):1024-1040. https://doi.org/10.1037//0003066x.57.12.102

34. Cluver L, Orkin M, Boyes M, Gardner F, Meinck F. Transactional sex amongst AIDS-orphaned and AIDS-affected adolescents predicted by abuse and extreme poverty. J Acquir Immune Defic Syndr 2011;58(3):336-343. https://doi.org/10.1097/qai.0b013e31822fod82

35. Cluver LD, Orkin M, Gardner F, Boyes ME. Persisting mental health problems among AIDS-orphaned children in South Africa. J Child Psychol Psychiatry 2012;53(4):363-370. https://doi.org/10.1111/ j.1469-7610.2011.02459

36. Mellins CA, Malee KM. Understanding the mental health of youth living with perinatal HIV infection: Lessons learned and current challenges. J Int AIDS Soc 2013;16(1):1-19. https://doi.org/10.7448/

37. Li X, Naar-King S, Barnett D, Stanton B, Fang X, Thurston C. A developmental psychopathology framework of the psychosocial needs of children orphaned by HIV. J Assoc Nurses AIDS Care 2008;19(2):147-157. https://doi.org/10.1016/j.jana.2007.08.004

38. South Africa. Children's Act No. 38 of 2005.

39. South Africa. Choice on Termination of Pregnancy Act No 92 of 1996

40. Flicker S, Guta A. Ethical approaches to adolescent participation in sexual health research. J Adolesc Health 2008;42(1):3-10. https://doi.org/10.1016/ji.jadohealth.2007.07.017

41. Caffey J. Multiple fractures in the long bones of infants suffering from chronic subdural hematoma. Am J Roentgenol Radium Ther 1946;56(2):163-173.
42. Weithorn LA. Children's capacities to decide about participation in research. IRB Ethics Hum Res 1983;5(2):1-5. https://doi.org/10.2307/3563792

43. Slack C, Strode A, Fleischer T, Gray G, Ranchod C. Enrolling adolescents in HIV vaccine trials: Reflections on legal complexities from South Africa. BMC Med Ethics 2007;8(1):5-13. https://doi. Reflections on legal complex

44. Langhinrichsen-Rohling J, Arata C, O’Brien N, Bowers D, Klibert J. Sensitive research with adolescents: Just how upsetting are self-report surveys anyway? Violence Vict 2006;21(4):425-444. adolescents: Just how upsetting are
https://doi.org/10.1891/vivi.21.4.425

45. Devries KM, Child JC, Elbourne D, Naker D, Heise L. 'I never expected that it would happen, coming to ask me such questions'. Ethical aspects of asking children about violence in resource poor settings. to ask me such questions': Ethical aspects of asking children about vione
Trials 2015;16(1):516-528. https://doi.org/10.1037/e500792015-293

46. Thurman TR, Snider L, Boris N, et al. Psychosocial support and marginalization of youth-headed households in Rwanda. AIDS Care 2006;18(3):220-229. https://doi.org/10.1080/09540120500456656

47. Thurman TR, Brown L Richter L Maharaj P, Magnani R. Sexual risk behavior among South African adolescents: Is orphan status a factor? AIDS Behav 2006;10(6):627-635. https://doi.org/10.1007 adolescents: Is orp

48. Naidoo P, Donenberg G, Davids A, et al. Exploring risk and protective mechanisms associated with HIV infection among adolescents in South Africa. J Psychol Afr 2014;24(3):232-240.

49. Kirby M. The never-ending paradoxes of HIV/AIDS and human rights. Afr Hum Rights Law 2004;4(2):163-180.

50. Strode A, Richter M, Wallace M, Toohey J, Technau K. Failing the vulnerable: Three new consent norms that will undermine health research with children. South Afr J HIV Med 2014;15(2):46-49. https://doi.org/10.7196/sajhivmed.1014

51. South Africa. Family Violence Act No. 133 of 1992

52. South Africa. Criminal Law (Sexual Offences and Related Matters) Amendment Act No. 32 of 2007. 53. Clacherty G, Donald D. Child participation in research: Reflections on ethical challenges in the souther African context. Afr J AIDS Res 2007;6(2):147-156. https://doi.org/10.2989/16085900709490409

54. Coad J. Using art-based techniques in engaging children and young people in health care consultation and/or research. J Res Nurs 2007;12(5):487-497. https://doi.org/10.1177/1744987107081250

55. D’Amico M, Denov M, Khan F, Linds W, Akesson B. Research as intervention? Exploring the health and well-being of children and youth facing global adversity through participatory visual methods. Glob Public Health 2016;11(5-6):528-545. https://doi.org/10.1080/17441692.2016.1165719

56. Willis N, Frewin L, Miller A, Dziwa C, Mavhu W, Cowan F. 'My story' - HIV positive adolescents tell their story through film. Child Youth Serv Rev 2014;45:129-136. https://doi.org/10.1016/j. childyouth.2014.03.029

57. Gorbach PM, Mensch BS, Husnik M, et al. Effect of computer-assisted interviewing on self-reported sexual behavior data in a microbicide clinical trial. AIDS Behav 2013;17(2):790-800. https://doi. org/10.1007/s10461-012-0302-2

58. Toska E, Cluver LD, Hodes R, Kidia KK. Sex and secrecy: How HIV-status disclosure affects safe sex among HIV-positive adolescents. AIDS Care 2015;27(Suppl 1):47-58. https://doi.org/10.1080/09540 121.2015.107177

59. Pettifor A, Bekker LG, Hosek S, et al. Preventing HIV among young people: Research priorities fo the future. J Acquir Immune Defic Syndr 2013;63(Suppl 2):S155-S160. https://doi.org/10.1097 qai.0b013e31829871f

60. Kieling C, Baker-Henningham H, Belfer M, et al. Child and adolescent mental health worldwide Evidence for action. Lancet 2011;378(9801):1515-1525. https://doi.org/10.1016/s0140-6736(11)60827-1

Accepted 5 April 2017. 